\title{
Isolation and characterization of Streptomyces species against vancomycin resistant enterococcus (VRE) from agricultural soils of Qom, Iran
}

\author{
Fatemeh Alzamoli
}

Islamic Azad University, Qom Branch, Iran

\begin{abstract}
Infections due to Vancomycin Resistant Enterococcus (VRE) are increasing worldwide. Resistance of VRE to glycopeptides antibiotics is related to van A,B,C,D,E genes which decreases the impact of this antibiotics on the cell wall of bacteria , making these bacteria innately Tuberamycin-resistant. Therefore, Jentamicin is used to treat infections. In recent years it has been reported that many of VRE are showing high resistance to Aminoglycosids and even to Jentamycin. There have been reports on distribution of VRE strains in clinical environments. This research was done to obtain novel antimicrobial agents which is effective against VRE infections. Enterococcus mainly cause nosocomial infections especially in ICU and CCU sections of hospitals and are threat to patients. Soil samples were collected from agricultural soils of Qom. Then isolation and primary identification of Streptomycetes was done on ISP4 culture medium. Then the antimicrobial activity of all Streptomycetes obtained were examined against VRE on Hickey-Trenser medium, Brain-heart infusion medium, Muller-Hinton agar with cross-streak method. Five out of 125 isolates showed inhibitory effect on VRE. In the next step the Streptomycete Strain that left bigger inhibition zone against VRE on medium were selected to be characterized biochemically and morphologically and was named as E.P1. Additionally were identified on the basis of its 16S rRNA sequence. Identification results indicated that the obtained Streptomyces strain is 100\% similar to Streptomyces tendae ATCC 19812.Finally, GC-Mass analysis was done on the antimicrobial agent that was extracted by ethyl acetate. Results revealed that it was a quinuline compound.
\end{abstract}

KEY WORDS: STREPTOMYCES ISOLATION, VRE, SECONDARY METABOLITE

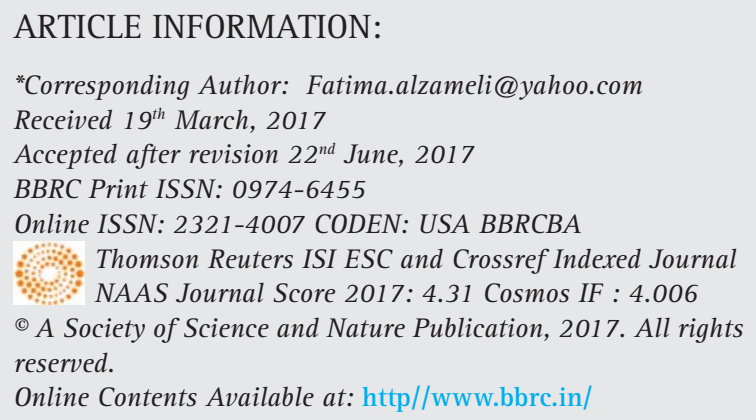




\section{INTRODUCTION}

Enterococcus can be resistant to different antibiotics. One of the main causes that distributes drug-resistant bacteria is routed in antibiotic misuse by communities. This new phenomenon causes severe infections and death of patients. Challenging problems of increasing drug-resistant bacteria urges the need to find more effective antibiotics for treatment (Yehuda et al, 2002). Antimicrobial producing microorganisms are distributed widely in nature and play key role in soil, water and degradation. Actinomycetes contains one of the largest of bacterial genera, Streptomyces known to be the source for many antibiotics. These are saprophytic,

\begin{tabular}{|c|c|}
\hline Streptomycetes isolates & Inhibition zone size $(\mathrm{mm})$ \\
\hline E.P1 & 26 \\
\hline
\end{tabular}

gram-positive that are major contributors to biological buffering of soils. This useful bacteria group produces secondary metabolites such as antibiotics, enzymes, immunoregulators, immunosupresores, antitumors and vitamins. These bacteria group play key part in organic compounds, food processing, pharmaceutical products, agriculture and fishing industry (Usha, 2010 and Mohitosh Biswas et al,2011).

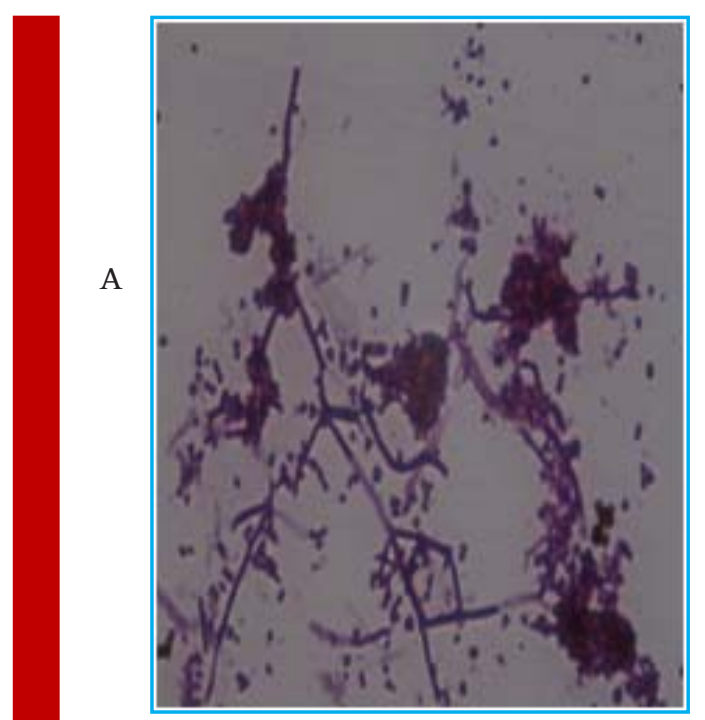

B

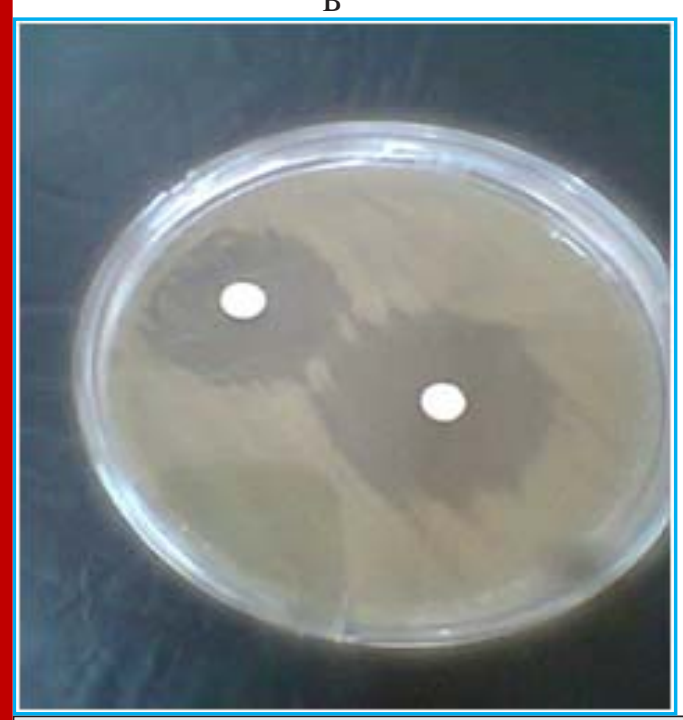

FIGURE 1. A:The inhibition zone on the right is made by the E.P1 isolate B: Gram staining of E.P1 isolate under the light microscopy C: Colony morphology of E.P1 isolate on ISP4 culture medium 


\section{MATERIAL AND METHODS}

One hundred and twenty five Streptomycetes were obtained from different sites in Qom. Soil samples were taken from 5-15 cm depth of soil and then were put in bags containing $0.1 \mathrm{~g}$ of caco3. Samples were incubated in 28 degree centigrade for about five days (Kavya et al., 2012 and Gurung et al., 2009).

Samples were diluted and $0.1 \mathrm{ml}$ of each sample were transferred to ISP4 culture medium, after that, were incubated for seven days in 28 degree centigrade for further study on isolates. Visual observation of both morphological and microscopic characteristics of isolates were done by colony investigation color and shape and using light microscopy.Those colonies that were identified as Streptomyces in previous stage were transferred to ISP2 agar incubated in 28 degree centigrade for up to seven days to obtain purified isolates for further study (Kavya, 2012).The standard isolate of VRE ATCC 51299 were brought from Reference Laboratory of Tehran.

The target Streptomycetes isolates were cultured on the center of Hicky-Trenser agar and then were incubated in 28 degree centigrade for 10 days. Next the Brain-Heart Infusion broth containing VRE was added to this medium to observe antimicrobial activity of every streptomycete isolate. The results were investigated after 24-48 hours (Alireza Dehnad et al., 2010). Biochemical test done on the target isolate of E.P1 includes: Melanin pigment production, Starch hydrolysis, Tween 80 hydrolysis, lecithinase test, Nitrate Broth, Oxidase test, Catalase test, DNase, Tryptophan hydrolysis, Acid Fast Staining and Lysozyme Resistance (Houssam, 2009). Antimicrobial producing Streptomyces E.P1 was cultured on ISP4 salnt medium in tube and incubated at 28 degree Centigrade for 10 days. Then spore suspension was provided. Firstly, $10 \mathrm{ml}$ of sterile water was added to slant medium and

\begin{tabular}{|l|l|}
\hline \multicolumn{2}{|l|}{ Table 2. Biochemical test results of } \\
Streptomyces E.P1 isolate \\
\hline Biochemical tests & E.P1 isolate \\
\hline Melanin Pigmentation & + \\
\hline Starch hydrolysis & + \\
\hline Tween 80 hydrolysis & - \\
\hline Licethinase test & + \\
\hline Nitrat test & - \\
\hline Oxidase test & - \\
\hline Catalase test & + \\
\hline DNase test & - \\
\hline Tryptophan hydrolysis & + \\
\hline Acid fast staining & - \\
\hline Lysosyme resistance & - \\
\hline
\end{tabular}

E.P1 colonies scratched. Secondly, $1 \mathrm{ml}$ of the suspension was transferred to fermentation erlen containing $50 \mathrm{ml}$ of culture medium. This erlen was incubated in shaker at 150,170,200 rpm in 28 degree centigrade for 14 days. Then $1 \mathrm{ml}$ of fermentation medium was centrifuged at $5000 \mathrm{rpm}$ for $15 \mathrm{~min}$ to remove traces of fermentation broth. Finally 20 microliter from supernatant was added to the center of Muller-Hinton agar in which VRE was cultured on previously. This plate was incubated at 30 degree centigrade and the inhibition zone was observed and measured (Osman et al.,2011).

Ethyl acetate solvent was used for the extraction of the antimicrobial from culture supernatant. The solvent was centrifuged at $5000 \mathrm{rpm}$ for $15 \mathrm{~min}$ to remove traces of fermentation broth. The extracted antimicrobial substance was identified by Gas-ChromatographyMass Spectrometry (GC-MS) analysis (Naggar et al., 2006).

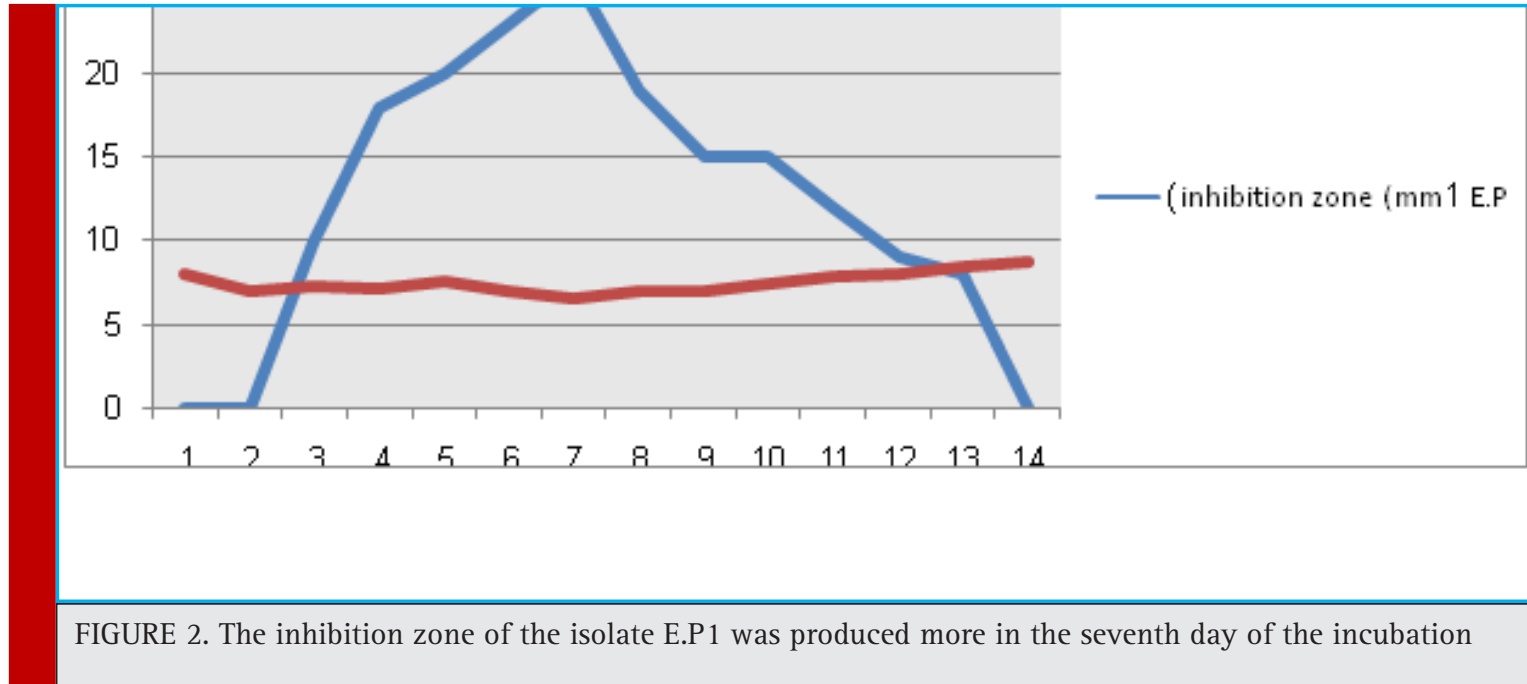




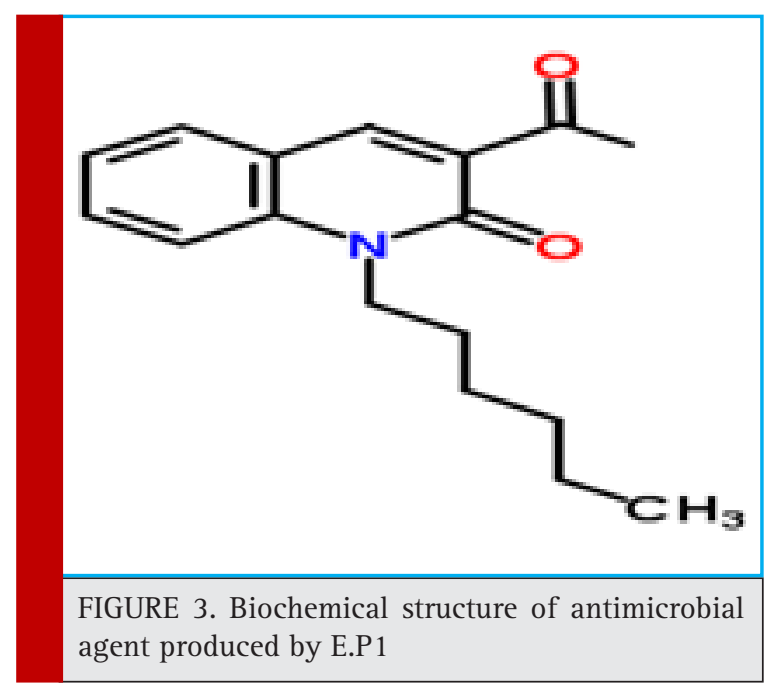

\section{RESULTS AND DISCUSSION}

Five Streptomycetes isolates could inhibited the growth of VRE. The isolate that showed the strongest inhibitory effect (E.P1) was chosen to be characterized .
After careful investigation of the result of GC-MS analysis of the antimicrobial compound it was named as: 3-Quinoline Carboxylic acid,1-hexyl-1,2-dihydro2-oxo

The $16 \mathrm{~s}$ rRNA sequence of Streptomyces E.P1 isolated showed 100\% similarity to Streptomyces tendae. Moreover, antimicrobial agent obtained from the target isolate is a quinoline compound.

There were similar studies done in this area and different results obtained. Rhee et al., attempted to isolate an Actinomycete that inhibited the growth of VRE in 2002 in Korea. They were successful to isolate a potential Actinomycete strain KH-614 against VRE that produced antitumor metabolite, too (Rhee et al., 2002).

El-Naggar could isolate Streptomyces HAL64 from Eygept soils that produced antimicrobial agent against gram-positive pathogens but not effective against gram-negative pathogens. He could extract the obtained antimicrobial substance by silicagel and then was purified with Sephadex LH-20 column. Finally HPLC was done to prove the purification of the antimicrobial agent (El-Naggar., 2007).

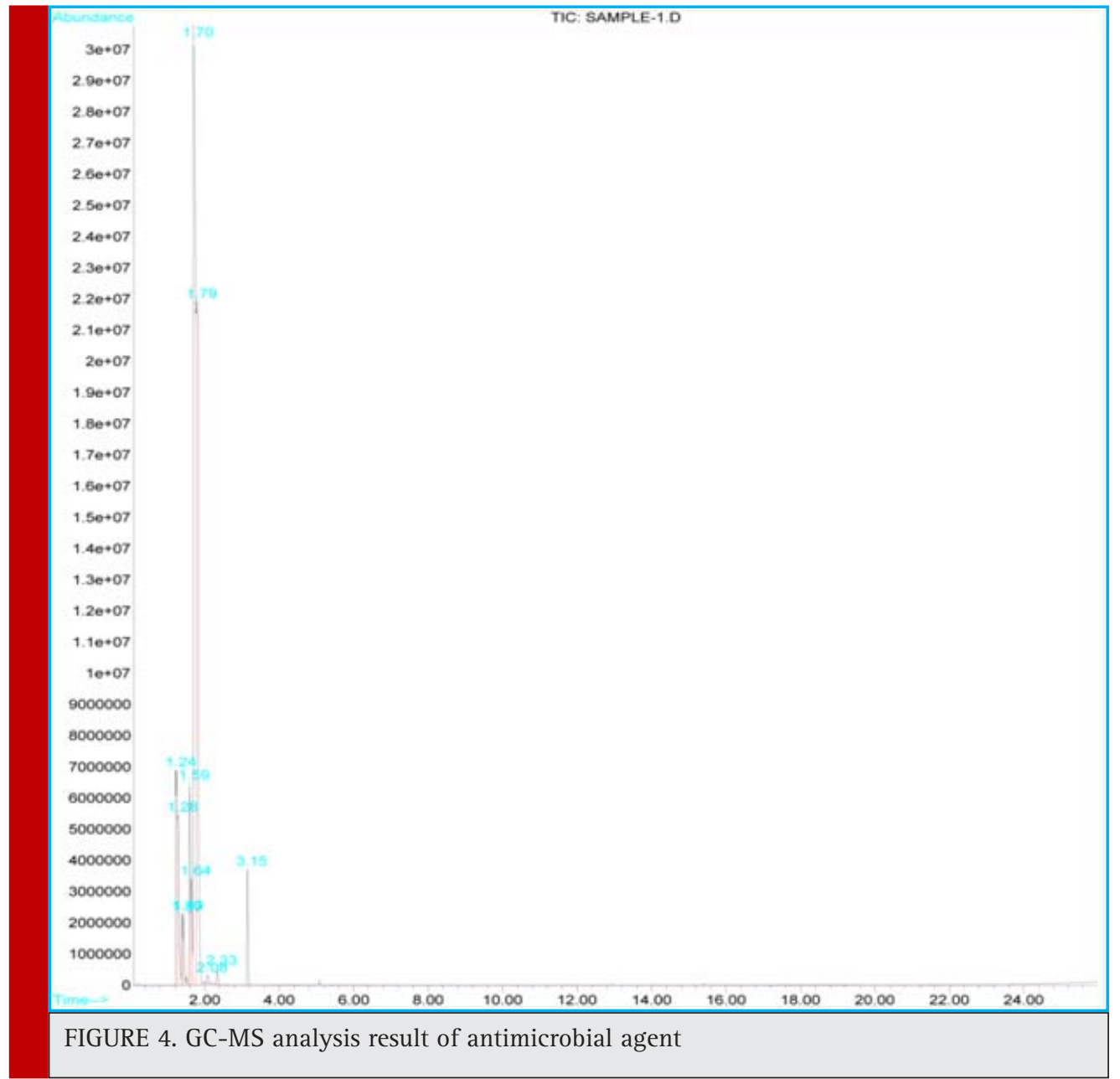




\section{ACKNOWLEDGEMENT}

The author is thankful to professor Ahmad Ali Pourbabaei (Lecturer at Tehran University, Iran) and Dr Seyyed Soheil Aghaei (Lecturer at Azad University of Qom, Iran).

\section{REFERENCES}

Alireza Dehnad, Lalle Parsa, Rouhollah Bakhshi, Samad Abdi Soofiani and Ahad Mokhtarzadeh, 2010. Investigating antibacterial activity of Streptomycetes isolates from soil samples, West of Iran. African Journal of Microbiology Research vol. 4(14), pp: 1542 - 1549.

El-Naggar, M.Y. 2007. Kosinostatin, a Major Secondary Metabolite Isolated from the Culture Filtrate of Streptomyces violaceusniger Strain HAL64. The Journal of Microbiology. 45(3), 262-267.

Fischett, V and Ryan, P. The genus Streptococcus. Chapter 23. In: Goldman, E, and Green, L, editors. 2th ed. Taylor \&t Francis Group, New York, 2009. 295-307.

Houssan M. Atta, 2009. An Antifungal Agent Produced by Streptomyces olivaceiscleroticus, Az - SH 514. World Applied Science Journal 6 (11): 1495 - 1505.

Ki Hyeong Rhee, 2002. Isolation and characterization of Streptomyces sp. KH - 614 Producing anti VRE (Vancomycin Resistant entrococci) antibiotics. J. Gen. Appl. Microbial. ,48: $321-327$.

Kavya Deepthi, M. Solomon Sudhakar and M. Nagalakshmi Devamma, 2012, Isolation and Screening of Streptomyces sp.
From coringa Mangrove soils for Enzyme production and Antimicrobial activity. IJP CBS, 2 (1): 110 - 116.

Mohammad E. Osman Fath Allah H. Ahmad and Walla S. M. Abd El All, 2011. Antibiotic production from Local Streptomyces Isolates from Egyptian soil at Wedey El Natron. Australian journal of Basic and Applied Sciences, 5: 1906 - 1910.

Mohitosh Biswas, Md. Ajijur Rahman, MST. Hejera Khatun and Md. Anwar - ul Islam, 2011. Isolation and characterizations of Streptomyces sp. ANBS - 15 and Antimicrobial Activities of its secondary Metabolies. Bangladesh Pharmaceutical Journal, Vol. 14, No. 1. p: 15 - 20.

Mostafa Y. El - Naggar, Samy A. El - Assar and sahar M. Abdul - Gawad, 2006. Meroparamycin production by newly Isolated Streptomyces sp. Strain MARO 1: Taxonomy, Fermentation, Purification and structural Elucidation. The journal of Microbiology, p: 432 - 438.

Tara Devi Gurung, Chringma Sherpa, Vishwanath Prasad Agrawal and Binod Lekhak, 2009. Isolation and characterization of Antibacterial Actinomycetes from soil samples of Kalapathar Mount Everest Region. Nepal Journal of science and Technology 10, pp: $173-182$.

Usha, P., Ananthaselvi, P., Venil, C and Palaniswamy, M. 2010. Antimicrobial and Antiangiogenesis activity of Streptomyces parvulus KUAP106 from Mangrove soil. European Journal of Biological Sciences. 2(4), 77-83.

Yehude Carmeli, George M. Eliopouloos, and Matthew H. Samore, 2002. Antecedent treatment with different Antibiotic Agents as a risk factor for vancomycin Resistant Enterococcus. Emerging Infections Diseases. Vol. 8: 802 - 809. 